\title{
Feasibility of Performing Sentinel Lymph Node Biopsy (SLNB) after Mastectomy: A Case Report
}

\author{
Hanadi Bu-Ali ${ }^{1}$, Eleftherios P. Mamounas ${ }^{2}$ \\ ${ }^{1}$ Aultman Cancer Center, Canton, $\mathrm{OH}$ and Akron General Medical Center, Akron, USA; ${ }^{2}$ Aultman Cancer Center, Canton, OH and \\ Northeastern Ohio Universities College of Medicine, Rootstown, USA. \\ Email: drhan@hotmail.com
}

Received April 11 ${ }^{\text {th }}, 2010$; revised May $2^{\text {nd }}, 2010$; accepted May $24^{\text {th }}, 2010$.

\begin{abstract}
Introduction: Previous mastectomy remains a contraindication to SLNB as normal drainage patterns of the breast can be disturbed. Patients diagnosed with DCIS on core biopsy and later found to have microinvasive or invasive carcinoma at the time of mastectomy are deprived of the opportunity for SLNB and need to undergo axillary dissection. We explored the option and feasibility of performing SLNB in a 39-year-old female who underwent a simple mastectomy without axillary sampling for extensive DCIS and later found to have microinvasive ductal carcinoma on permanent pathology. Results: Lymphatic mapping using subdermal injection of $99 \mathrm{mTc}$-labeled sulfur colloid and blue dye led to the identification of five SLNs. Histopathologic examination showed no metastasis. Conclusion: SNLB is feasible in this setting. However, before its use is routinely adopted, its feasibility and accuracy has to be demonstrated in larger numbers of patients in whom a negative SLNB is followed by a completion axillary dissection.
\end{abstract}

Keywords: Sentinel Lymph Node Biopsy, Lymphatic Mapping, Mastectomy, Lymphoscintigraphy, Axillary Lymph Node Dissection

\section{Introduction}

Axillary lymph node status remains the single most important prognostic factor for invasive, early-stage breast cancer. Currently, SLNB is routinely performed in clinical practice but the indications for SLNB continue to evolve. In the 1990s, SLNB was strictly limited to patients with small invasive tumors and clinically negative axillae. With increased experience and the widespread use of the technique, indications for SLNB have since expanded to most patients with clinically node-negative, operable breast cancer. More recently, the suitability of SLNB has also been considered in the setting of intraductal carcinoma (DCIS) and DCIS with microinvasion. However, previous mastectomy remains a contraindication to SLNB as it has been suggested that the normal drainage patterns of the breast can be disturbed shortly after surgery or radiotherapy and that prior excisional biopsy can alter lymphatic drainage in up to $70 \%$ of the patients. Thus, patients who are diagnosed with DCIS on core needle biopsy and are later found to have microinvasive or invasive carcinoma after mastectomy are deprived of the opportunity for SLNB and need to undergo an axillary dissection. We evaluated the feasibility of SLNB in a patient who was referred to our center fol- lowing simple mastectomy for a large area of DCIS (based on core biopsy) and who was later found to have microinvasive breast cancer on permanent pathology.

\section{Case Report}

A 39-year-old Caucasian female underwent a simple mastectomy for extensive DCIS, one month prior to presentation to our center. No histologic examination of the axilla was performed. She was found to have microinvasive ductal carcinoma on permanent pathology and was recommended to undergo axillary dissection but wanted to explore alternatives, including SLNB. We proceeded with lymphatic mapping and SLNB. Lymphatic mapping was performed using subdermal injections of $99 \mathrm{~m}$ Tc-labeled sulfur colloid in the skin flaps both above and below the previous mastectomy scar. Subdermal injections of blue dye took place intraoperatively in the same fashion and the area was massaged for five minutes. Axillary lymph nodes were designated as sentinel nodes (SLN) and biopsied if they were clinically palpable in surgery, radioactive, blue or any combination of the above.

Five SLNs were identified. One was hot (10-second count of 169), one was blue but not hot and three were 
only clinically palpable but not clinically suspicious. Histopathologic examination showed no metastasis. Given the low-risk for axillary metastasis, no completion axillary dissection was performed.

\section{Discussion}

Lymphatic mapping and SLNB are well-established tools for staging primary breast carcinoma and minimizing morbidity associated with level I and II axillary node dissection (ALND). Axillary SLNB is currently considered the gold standard for staging the axilla in clinically node-negative women [1]. Several investigators have confirmed the hypothesis that lymphatic drainage of a breast cancer can be identified and traced to the sentinel node during surgery, and that the histologic status of the sentinel node accurately predicts the pathologic status of the entire axilla [2,3-9]. The sentinel lymph node concept for early breast cancer has been well validated in many studies. [4,5,7-9,10-12] Although initially lymphatic mapping was performed by peritumoral injections of radioisotope or blue dye, more recently, the intradermal injection route has been shown to result in a significantly greater frequency of localization, decreased time to first localization on preoperative lymphoscintigraphy, and decreased time to harvest the first SLN [13].

To be anatomically and oncologically effective and to correctly predict the histological status of the axilla, SLNB requires the presence of an intact lymphatic flow from the site of the primary tumor (or recurrence). Previous mastectomy partially and/or temporarily interrupting and modifying the lymphatic flow renders it theoretically more difficult to correctly identify the SLN. In such a situation the concept of SLN itself could be questioned and ALND is always considered mandatory, even in cases of small limited recurrences with low risk of axillary involvement. Thus, following mastectomy, several questions need to be addressed relative to the potential value of pursuing a SLNB. Where is the chest wall lymphatic drainage anatomically directed? And if a new SLN exists, is its predictive value biologically comparable to that of SLNs from an intact breast? [14]

The breast drains through common afferent lymphatic channels to common axillary nodes [15]. This lymphatic pathway supports the hypothesis that the breast drains as a single unit, and therefore the location of the primary tumor within the breast does not affect the location and number of the sentinel nodes with the exception of tumors located deep in the breast, which can drain either to the axilla or to the internal mammary chain via the deep retromammary lymphatic pathway.[16] Thus, the variable which determines the number and location of SLNs in the axilla is the number of afferent lymphatic pathways to the axilla rather than the number or site of injections. This evolution in the SLN concept has also been considered the rational basis on which SLNB may be applied to patients with a multicentric disease [17]. A mastectomy can interrupt and strongly modify the lymphatic flow of the thoracic wall, but the physiological restoring of the anatomy of the lymphatic drainage renders the obstacle only temporary. In fact, when an adequate period of time elapses between the mastectomy and the local recurrence, the lymphatic network has time to be physiologically re-built and a new lymphatic "bridge" can connect the thoracic wall to the afferent loco- regional lymph nodes. Such a postoperative "collateralization" of lymphatics occurs as a compensatory mechanism. Presumably, the once predictable nodal drainage architecture becomes distorted, but this need not necessarily preclude the presence of a loco-regional SLN in such cases. In fact, a novel SLN has been documented in a case of small breast recurrence after breast conserving surgery (BCS) and complete axillary dissection (CAD) (the new SLN was in the contralateral axilla) [18], after partial axillary dissection (PAD) (the new SLN was in the ipsilateral axilla) [19], and after previous SLNB (again, the new SLN was in the ipsilateral axilla) [20]. These considerations, introducing a new dynamic concept of SLN (not "one SLN for ever" but "always a new SLN"), have convinced authors to propose and successfully perform lymphoscintigraphy and SLNB after BCS and previous CAD [18], PAD [19] and SLNB [20].

In women with a recurrent or second ipsilateral breast carcinoma and history of previous breast and axillary surgery lymphoscintigraphy is successful in identifying a SLN in $69 \%$ of patients. Lymphatic drainage patterns -including drainage across the midline of the thorax in $25 \%$ of patients - vary widely. Lymphoscintigraphy in these patients is especially useful for preoperative planning to ensure inclusion of potential sentinel nodes in the contralateral axilla or supraclavicular and infraclavicular regions within the operative field. Although internal mammary nodes are not routinely biopsied, identification of internal mammary nodes may be useful for prognostication and radiation treatment planning1. Using dual tracers consisting of blue dye and lymphoscintigraphy with technetium radiocolloid affords surgeons an opportunity to observe actual native and collateralized lymph node drainage architecture in real-time. Subsequent identification of nodal uptake in any nodal region for patients with primary or recurrent breast malignancies may provide pertinent clinical data relevant to optimal staging and treatment [18]. The same theory of lymphatic dynamics following BCS for patients with primary or recurrent breast malignancies could be applied for patients following mastectomy. While there is paucity in the literature of previous experiences with SLNB following mastectomy, the same concept of lymphatic distortion and, hence, the same potential challenges with SLNB apply.

\section{Conclusions}

Subdermal injection of both radioisotope and blue dye led to the identification of axillary SLNs in a patient who 
had recently undergone a simple mastectomy. This case demonstrates the potential feasibility of the procedure in this setting. However, before its use is routinely adopted, its feasibility and accuracy has to be demonstrated in larger numbers of patients in whom a negative SLNB is followed by a completion axillary dissection.

\section{REFERENCES}

[1] D. Dinan, C. E. Nagle, J. Pettinga, "Lymphatic Mapping and Sentinel Node Biopsy in Women with an Ipsilateral Second Breast Carcinoma and a History of Breast and Axillary Surgery," AJS, Vol. 190, 2005, pp. 614-617.

[2] A. D. K. Hill, K. N. Tran, T. Akhurst, H. Yeung, S. D. J. Yeh, P. P. Rosen, P. Borgen and H. S. Cody, "Lessons Learned from 500 Cases of Lymphatic Mapping for Breast Cancer," Annals of Surgery, Vol. 229, No.4, April 1999, pp. 528-535.

[3] A. E. Giuliano, D. M. Kirgan, J. M. Guenther and D. L. Morton, "Lymphatic Mapping and Sentinel Lymphadenectomy for Breast Cancer," Annals of Surgery, Vol. 220, 1994, pp. 391-401.

[4] A. E. Giuliano, R. C. Jones, M. Brennan and R. Statman, "Sentinel Lymphadenectomy in Breast Cancer," Journal of Clinical Oncology, Vol. 15, 1997, pp. 2345-2350.

[5] U. Veronesi, G. Paganelli, V. Galimberti, et al., "Sentinel Node Biopsy to Avoid Axillary Dissection in Breast Cancer with Clinically Negative Nodes," Lancet, Vol. 349, 1997, pp. 1864-1867.

[6] A. E. Giuliano, A. M. Barth, B. Spivack, P. D. Beitsch, S. W. Evans, "Incidence and Predictors of Axillary Metastases in T1 Carcinoma of the Breast," Journal of American College Surgeons, Vol. 183, 1996, pp. 185-189.

[7] D. N. Krag, T. Ashikaga, S. P. Harlow, D. L. Weaver, "Development of Sentinel Node Targeting Techniques in Breast Cancer Patients," Breast Journal, Vol. 4, 1998, pp. 67-74.

[8] J. J. Albertini, G. H. Lyman, C. Cox, et al., "Lymphatic Mapping and Sentinel Node Biopsy in the Patient with Breast Cancer," Journal of the American Medical Association, Vol. 276, 1996, pp. 1818-1822.

[9] P. J. Borgstein, S. Meijer, R. Pijpers, "Intradermal Blue Dye to Identify Sentinel Lymph-Node in Breast Cancer," Lancet, Vol. 349, 1997, pp. 1668-1669.

[10] J. M. Guenther, M. Krishnamoorthy, L. R. Tan, "Sentinel lymphadenectomy for Breast Cancer in a Community Managed Care Setting," Cancer Journal, Vol. 3, 1997, pp. 336-340.
[11] S. Meijer, G. J. Collet, H. J. Pijpers, L. van Hattum, O. S. Hoekstra, "Less Axillary Dissection Necessary Due to Sentinel Node Biopsy in Patients with Breast Carcinoma," Nederlands Tijdschrift voor Geneeskunde, Vol. 140, 1996, pp. 2239-2243.

[12] O. E. Nieweg, B. A. Kapteijn, J. L. Peterse, E. J. Rutgers, J. A. van Dongen and B. B. Kroon, "Identification of the Sentinel Node in Patients with Breast Carcinoma," Nederlands Tijdschrift voor Geneeskunde, Vol. 140, 1996, pp. 2235-2239.

[13] S. P. Povoski, J. O. Olsen, D. C. Young, J. Clarke, W. E. Burak, M. J. Walker, W. E. Carson, L. D. Yee, D. M. Agnese, R. V. Pozderac, N. C. Hall and W. B. Farrar, "Prospective Randomized Clinical Trial Comparing Intradermal, Intraparenchymal, and Subareolar Injection Routes for Sentinel Lymph Node Mapping and Biopsy in Breast Cancer," Annals of Surgery Oncology, Vol. 13, No. 11, November 2006, pp. 1412-1421.

[14] M. Intra, P. Veronesi, O. D. Gentilini, G. Trifiro, A. Berrettini, R. Cecilio, M. Colleoni, M. Rietjens, A. Luini, G. Paganelli, U. Veronesi, "Sentinel Lymph Node Biopsy Is Feasible Even after Total Mastectomy," Journal of Surgical Oncology, Vol. 95, 2007, pp. 175-179.

[15] T. M. Tuttle, M. Colbert, R. Christensen, et al., "Subareolar Injection of 99mTc Facilitates Sentinel Lymph Node Identification," Annals of Surgery Oncology, Vol. 9, 2002, pp. 77-81.

[16] K. Shimazu, Y. Tamaki, T. Taguchi, et al., "Lymphoscintigraphic Visualization of Internal Mammary Nodes with Subtumoral Injection of Radiocolloid in Patients with Breast Cancer," Annals of Surgery, Vol. 237, 2003, pp. 390-398.

[17] E. Tousimis, K. J. van Zee, J. V. Fey, et al. "The Accuracy of Sentinel Lymph Node Biopsy in Multicentric and Multifocal Invasive Breast Cancers," Journal of American College Surgeons, Vol. 197, 2003, pp. 529-535.

[18] A. Agarwal, D. E. Heron, J. Sumkin, et al., "Contralateral Uptake and Metastases in Sentinel Lymph Node Mapping for Recurrent Breast Cancer," Journal of Surgical Oncology, Vol. 92, 2005, pp. 4-8.

[19] E. R. Port, J. Fey, M. L. Gemignani, et al., "Reoperative Sentinel Lymph Node Biopsy: A New Option for Patients with Primary or Locally Recurrent Breast Carcinoma," Journal of American College Surgeons, Vol. 195, 2002, pp. 167-172.

[20] M. Intra, G. Trifiro, G. Viale, et al., "Second Biopsy of Axillary Sentinel Lymph Node for Reappearing Breast Cancer after Previous Sentinel Lymph Node Biopsy," Annals of Surgery Oncology, Vol. 12, 2005, pp. 895-899. 